\title{
Exchange anisotropy as a probe of antiferromagnetism in expanded face-centered-tetragonal $\mathrm{Mn}(001)$ layers
}

\section{Citation for published version (APA):}

Kohlhepp, J. T., Wieldraaijer, H., \& Jonge, de, W. J. M. (2006). Exchange anisotropy as a probe of antiferromagnetism in expanded face-centered-tetragonal Mn(001) layers. Applied Physics Letters, 89(3), 032507-1/3. [032507]. https://doi.org/10.1063/1.2222342

DOI:

$10.1063 / 1.2222342$

Document status and date:

Published: 01/01/2006

\section{Document Version:}

Publisher's PDF, also known as Version of Record (includes final page, issue and volume numbers)

\section{Please check the document version of this publication:}

- A submitted manuscript is the version of the article upon submission and before peer-review. There can be important differences between the submitted version and the official published version of record. People interested in the research are advised to contact the author for the final version of the publication, or visit the $\mathrm{DOI}$ to the publisher's website.

- The final author version and the galley proof are versions of the publication after peer review.

- The final published version features the final layout of the paper including the volume, issue and page numbers.

Link to publication

\section{General rights}

Copyright and moral rights for the publications made accessible in the public portal are retained by the authors and/or other copyright owners and it is a condition of accessing publications that users recognise and abide by the legal requirements associated with these rights.

- Users may download and print one copy of any publication from the public portal for the purpose of private study or research.

- You may not further distribute the material or use it for any profit-making activity or commercial gain

- You may freely distribute the URL identifying the publication in the public portal.

If the publication is distributed under the terms of Article 25fa of the Dutch Copyright Act, indicated by the "Taverne" license above, please follow below link for the End User Agreement:

www.tue.nl/taverne

Take down policy

If you believe that this document breaches copyright please contact us at:

openaccess@tue.nl

providing details and we will investigate your claim. 


\title{
Exchange anisotropy as a probe of antiferromagnetism in expanded face-centered-tetragonal $\mathrm{Mn}(001)$ layers
}

\author{
J. T. Kohlhepp, ${ }^{\text {a) }}$ H. Wieldraaijer, and W. J. M. de Jonge \\ Department of Applied Physics, Eindhoven University of Technology, P.O. Box 513, 5600 MB Eindhoven, \\ The Netherlands and Center for NanoMaterials (cNM), Eindhoven University of Technology, P.O. \\ Box 513, 5600 MB Eindhoven, The Netherlands
}

(Received 20 March 2006; accepted 13 June 2006; published online 19 July 2006)

\begin{abstract}
Manganese (Mn) grows coherent and with an expanded metastable face-centered-tetragonal (e-fct) structure on ultrathin fct $\mathrm{Co}(001) / \mathrm{Cu}(001)$ template layers. From the temperature dependence of the observed unidirectional $\mathrm{Mn} / \mathrm{Co}$ interface exchange anisotropy, an antiferromagnetic state with a blocking temperature around $410 \mathrm{~K}$ is found for a 21 monolayer thick e-fct $\mathrm{Mn}(001)$ film. The temperature dependent coercivity enhancement of the Co films, which is induced by the proximity of the antiferromagnetic Mn layer, suggests a Néel temperature above $410 \mathrm{~K}$ for this Mn phase.

(C) 2006 American Institute of Physics. [DOI: 10.1063/1.2222342]
\end{abstract}

In the last two decades numerous research studies on magnetic exchange interactions observed between two magnetically ordered thin layers were reported. ${ }^{1,2}$ This tremendous interest not only stems from the intriguing fundamental physics but also from the importance of magnetic interactions for the functionality of modern so-called spintronic devices, which exploit the spin of an electron in addition to its electrical charge. ${ }^{3}$ Especially in giant magnetoresistance or tunnel magnetoresistance devices very often antiferromagnets (AFMs) exchange coupled to ferromagnets (FMs) are key components. Here, the function of an AFM is mainly to pin the magnetization of an adjacent FM to a certain direction to insure a desired switching behavior and ergo functionality. Another application could be envisioned in the field of future next generation high density recording media by exploiting the coercive field enhancements and magnetic anisotropy changes, which are typically observed in AFM/FM systems, to ensure the magnetic stabilization of nanometersize FM particles by surrounding them with an AFM shell. ${ }^{4}$

Despite this enormous potential for present and future technological applications, a fundamental understanding of the exact mechanisms, responsible for the so-called exchange anisotropy and several associated phenomena observed, ${ }^{1,2}$ is still not achieved. Mainly triggered by the direct application perspectives, the majority of the reported studies deals with polycrystalline samples. These structures, however, suffer from different sorts of imperfections, such as interdiffusion, uncontrolled interface roughness, grain size and phase distributions, random magnetic anisotropies, etc. Singlecrystalline systems with simple lattices offer well-defined and tunable interfaces, spin orientations, and anisotropies, allowing systematic studies to unveil in more detail the exchange mechanisms involved, but are only rarely reported. ${ }^{5,6}$

In this letter, we introduce a well-defined singlecrystalline AFM/FM model system which is based on tetragonally distorted $\mathrm{Mn}(001)$ thin films, epitaxially stabilized on fct $\mathrm{Co}(001)$. We present structural evidence for a metastable Mn phase, namely, expanded face-centered-tetragonal (e-fct), a slightly distorted $\mathrm{fcc}(\gamma)$ Mn phase with an axial

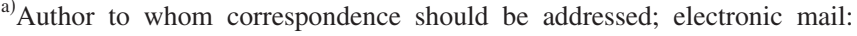
j.t.kohlhepp@tue.nl ratio $c / a>1$. Recent theoretical calculations have shown that, in addition to a contracted metastable phase $(\mathrm{c} / \mathrm{a}$ $=0.945)$, such a metastable structure with $c / a=1.048$ should indeed exist. ${ }^{7}$ By exploiting the exchange interaction at an $\mathrm{AFM} / \mathrm{FM}$ interface, it is shown that e-fct $\mathrm{Mn}(001)$ is in an AFM state with a Néel temperature $\left(T_{N}\right)$ well above room temperature.

The Mn layers were deposited on thin magnetic fct $\mathrm{Co}(001)$ template films, which were grown on $\mathrm{Cu}(001)$ single crystal substrates in a VG-Semicon V80M molecular beam epitaxy system. This multichamber system is equipped with a range of surface characterization techniques, such as scanning tunneling microscopy (STM), low energy electron diffraction (LEED), x-ray photoelectron spectroscopy (XPS), and Auger electron spectroscopy (AES). During the experiments it was noticed that the growth quality of the $\mathrm{Mn}$ is very sensitive to impurities. By using high purity Co (99.99\%) and Mn (99.999\%) and applying extensive outgassing procedures, deposition pressures below $5 \times 10^{-11} \mathrm{mbar}$ could be reached. In this way impurity inclusions in the films and at the interfaces were minimized. The $\mathrm{Cu}(001)$ crystals were treated with several Ar sputter and anneal cycles to ensure clean and atomically flat surfaces. During deposition the substrate temperature was maintained at $50{ }^{\circ} \mathrm{C}$. The $\mathrm{Mn}$ films reported here were grown at a rate of 1.5 monolayer $(\mathrm{ML}) / \mathrm{min}$ as deduced from an accurately calibrated quartz microbalance. After in situ characterization the $\mathrm{Co} / \mathrm{Mn}$ bilayers were covered with $20 \AA \mathrm{Cu}$ and $20 \AA \mathrm{Al}$ to avoid the oxidation of the magnetic layers in the ex situ magnetization measurements. These capping layers had no measurable influence on the structural properties of the Mn, at least not for the thicknesses we used for the ex situ experiments.

LEED diffraction patterns and LEED $I(V)$ curves of the 00 -spot were measured to identify the crystal structure and to determine the lattice constants. Complementary $\mathrm{x}$-ray photoelectron diffraction (XPD) and x-ray diffraction (XRD) experiments were used to confirm these results independently. The magnetic properties of the $\mathrm{Co} / \mathrm{Mn}$ bilayers were determined with the longitudinal magneto-optical Kerr effect (MOKE) at room temperature, and with a superconducting quantum interference device (SQUID) magnetometer working in the temperature range from 5 to $400 \mathrm{~K}$. While with 

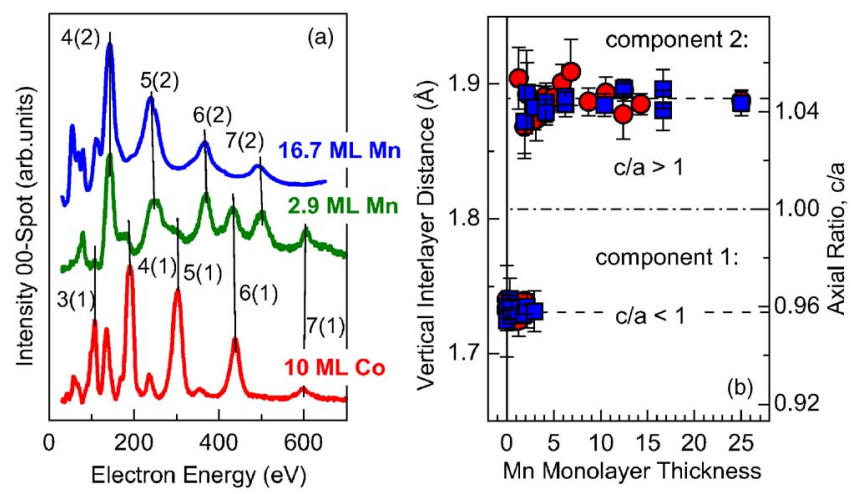

FIG. 1. (Color online) (a) LEED 00-spot $I(V)$ curves of $10 \mathrm{ML}$ $\mathrm{Co}(001) / \mathrm{Cu}(001)$ films, uncovered and covered with 2.9 and $16.7 \mathrm{ML}$ $\mathrm{Mn}(001)$, respectively. Depending on the Mn cover thickness two different series of Bragg peaks are observed as indicated by the two sets of maxima labeled (1) and (2). (b) Vertical interlayer distances deduced from such LEED $I(V)$ measurements vs the Mn overlayer thickness, either grown on $10 \mathrm{ML}$ (squares) or $20 \mathrm{ML}$ (circles) $\mathrm{Co}(001)$. The right axis shows the ratio of the out-of-plane to the in-plane lattice constant $(c / a)$ assuming coherent growth.

MOKE it was possible to rotate the external magnetic field along all desired crystal directions, in the SQUID, due to the limited sample space and the shape of the available $\mathrm{Cu}$ crystal, the field was restricted to the in-plane [100] $\mathrm{Cu}$ direction.

We have tested the growth of $\mathrm{Mn}$ on $\mathrm{Co}(001)$ template thicknesses of 10 and $20 \mathrm{ML}$. The thickness of $10 \mathrm{ML}$ is in the region where Co grows close to layer by layer on $\mathrm{Cu}(001)$ and adopts the $\mathrm{Cu}(001)$ in-plane lattice constant (pseudomorphic growth). For this thickness the Co(001) surface is dislocation-free and on average only two atomic levels are exposed, as seen by STM. ${ }^{8}$ For the second thickness, $20 \mathrm{ML}$, the Co starts to relax slightly, which is accompanied by the appearance of isolated dislocation lines and extra roughening of the surface (now exposing four atomic layers on average). ${ }^{8}$ However, the growth of Mn remains unaffected by these tiny but measurable changes of the Co structure and morphology. Initially, Mn fills the grooves on the Co surface and forms a very flat surface for the subsequent layer-bylayer growth mode of $\mathrm{Mn}$, which is observed up to a thickness of 10-15 ML (this thickness being slightly higher for the thinner and flater Co films). Beyond this, a somewhat roughened and disordered surface evolves. The LEED diffraction patterns of $\mathrm{Mn}$ overlayers is dominated by the existence of sharp $p(1 \times 1)$ spots at the positions of the Co substrate spots, demonstrating a coherent (pseudomorphic) growth with the Co in-plane interatomic spacing. However, in particular, for the growth on thin Co films, indications of several different surface reconstructions are observed. For example, between 2 and 4 ML a weak $c(12 \times 8)$ and above 10-15 ML a weak $p(18 \times 1)$ superstructure is visible. We interpret these reconstructions as being confined to the surface and not reflecting a major change in the bulk Mn structure. Such surface reconstructions are commonly found in the growth of metastable materials such as, e.g., fcc Fe(001) on $\mathrm{Cu}(001)$.

From LEED patterns we can determine the in-plane crystal symmetry and interatomic spacing, but no information about the lattice structure parallel to the surface normal is accessible. However, from the energy dependence of the intensity of the mirror beam ( 00 -spot), the vertical interlayer distance $d_{\perp}$ can be estimated by a simple kinematical Downloaded 13 Sep 2007 to 131.155.108.71. Redistribution subject

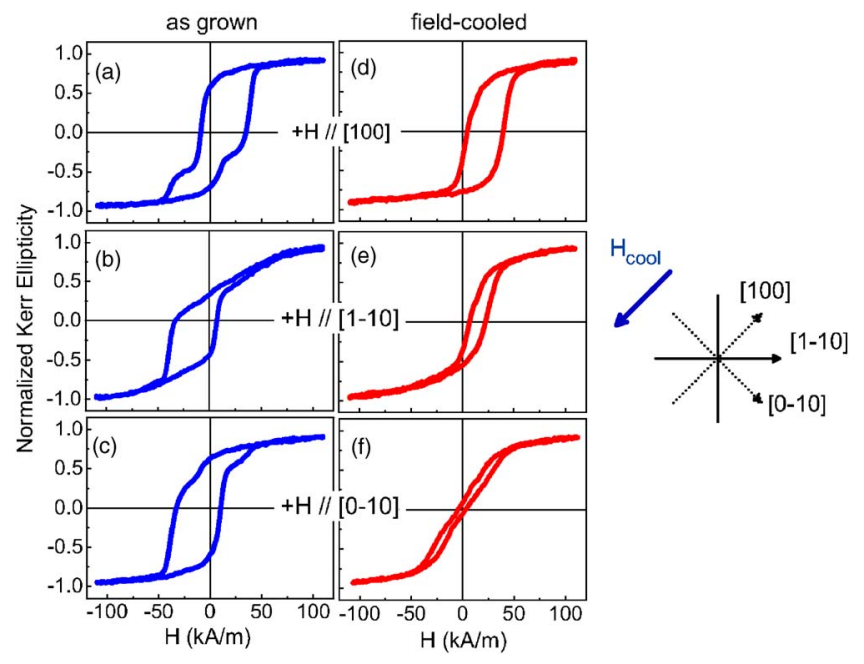

FIG. 2. (Color online) Room temperature longitudinal MOKE hysteresis loops of $21 \mathrm{ML} \mathrm{Mn} / 10 \mathrm{ML} \mathrm{Co} / \mathrm{Cu}(001)$ measured with an applied external field $H$ along different in-plane directions, both for as grown and from $T$ $=435 \mathrm{~K}$ field-cooled magnetization states. In the right cartoon the fieldcooling direction with respect to the crystal axes is depicted. Note that the MOKE laser spot did not stay exactly at the same sample position during rotation of the sample.

calculation. ${ }^{10}$ In Fig. 1(a) LEED $I(V)$ curves for uncovered films and those covered with 2.9 ML $\mathrm{Mn}(001)$ and 16.7 ML $\mathrm{Mn}(001)$ capped $10 \mathrm{ML} \mathrm{Co}(001) / \mathrm{Cu}(001)$ are plotted. Depending on the overlayer thickness, two different series of Bragg maxima are identified, and in a certain Mn thickness range (up to roughly $3.5 \mathrm{ML}$ ) a coexistence of both peak series is observed. We have performed $I(V)$ experiments for many different Mn overlayer thicknesses on both 10 and 20 ML thick Co films. The results of these experiments are shown in Fig. 1(b) and summarized as follows. While Bragg series (1) is clearly originating from diffraction at Co lattice planes, series (2) is unambiguously coming from the $\mathrm{Mn}$ lattice. In the coexistence regime electrons diffracted at the Co underlayer lattice are still detected through the Mn overlayer. This interpretation is supported by the observation that peaks of series (1) of Mn capped Co samples are distinctly more attenuated at lower order, which reflects the energy dependence of the inelastic mean free path of electrons originating from an underlayer (here Co) through an overlayer (here Mn). ${ }^{11}$ Series (1) is associated with $c=2 d_{\perp}=3.46 \AA$. Taking into account an ideal coherent growth of Co on $\mathrm{Cu}(001)$, this results in an axial ratio of $c / a=0.957$, in excellent agreement with earlier independent studies using other methods. ${ }^{12}$ For $\mathrm{Mn}$ [series (2)] we get $c=2 d_{\perp}$ $=3.77 \AA$, or alternatively an axial ratio of $c / a=1.043$, thus fct growth with an expanded $c$ axis (e-fct). This $c / a$ ratio is independent of both the Mn and the Co thicknesses used, indicating a metastable e-fct phase. Such a phase with $c / a$ $=1.048$ was actually predicted recently. ${ }^{7}$ Complementary XPD and XRD experiments resulting in $c / a=1.055$ have shown that the kinematic LEED probably slightly underestimates $c,{ }^{13}$ nevertheless all experiments unambiguously show that a metastable e-fct Mn phase indeed exists and that it can be stabilized on a suitable substrate such as, in our case, $\mathrm{Co}(001)$ coherently grown on $\mathrm{Cu}(001)$.

The specific layered structure which we used to stabilize e-fct $\mathrm{Mn}(001)$ on top of $\mathrm{Co}(001)$ offers also a unique possibility to trace the so far unknown magnetic characteristics of to AIP license or copyright, see http://apl.aip.org/apl/copyright.jsp 


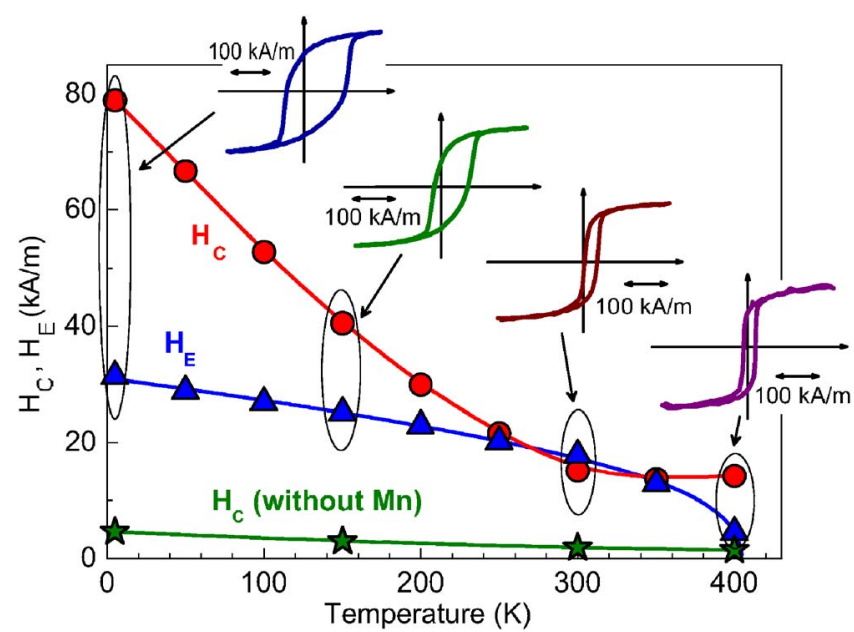

FIG. 3. (Color online) Temperature dependence of the coercive (circles) and exchange bias (triangles) fields of $21 \mathrm{ML} \mathrm{Mn} / 10 \mathrm{ML} \mathrm{Co} / \mathrm{Cu}(001)$ as measured by SQUID magnetometry. For comparison, the coercive fields of $\mathrm{Cu}$ covered $10 \mathrm{ML} \mathrm{Co} / \mathrm{Cu}(001)$ are shown (stars).

e-fct Mn. Thin fct Co(001) films are known to be ferromagnetic with $T_{C}$ 's above $600 \mathrm{~K}$ and well-defined magnetic anisotropies for thicknesses above 3-4 ML. ${ }^{14,15}$ These properties not only permit the detection of a potential AFM order in the Mn via the direct interface exchange coupling but also make the determination of temperature dependent AFM properties possible. An AFM order of e-fct $\mathrm{Mn}$ is indeed demonstrated by the MOKE magnetization loops depicted in Fig. 2. The as grown state, measured along three in-plane high-symmetry directions [Figs. 2(a)-2(c)], is characterized by multicomponent loops, reflecting the magnetic domain state of the as grown Co (frozen in by the Mn overgrowth) in the sample region probed by the laser spot. In all directions loop shifts caused by the pinning of the Co domains, which have magnetization directions along the $\langle 110\rangle$ easy axes, are observed. This is a clear sign of AFM/FM exchange interaction and thus AFM order in e-fct Mn. A homogeneous pinning direction is achieved by heating the sample above the blocking temperature $\left(T_{B}\right)$ of the AFM/FM system with a subsequent cooldown in a magnetic field. We choose the $[-100]$ direction because it is also the field direction available in our SQUID. The room temperature loops of the fieldcooled sample are plotted in Figs. 2(d)-2(f). They show that a well-defined exchange biased loop is observed when the field is applied along the field-cooling direction, whereas perpendicular to this direction a hard-axis loop is found.

The temperature dependence of the characteristic parameters of the exchange interaction, the coercive $\left(H_{C}\right)$ and exchange bias shift $\left(H_{E}\right)$ fields, are plotted in Fig. 3 for the same film as in Fig. 2. We utilized a 21 ML thick Mn layer because we have shown earlier, that this thickness is sufficient to approximate the "bulk" properties of e-fct $\mathrm{Mn}$ in which we are mainly interested in the present study. ${ }^{13}$ Thus, finite size effects will not play a role for this sample. Over the entire accessible temperature range, both distinct loop shifts and enhanced coercivities are detected. However, around $400 \mathrm{~K}$ a collapse of $H_{E}$ is observed. By extrapolation of $H_{E}$ to zero, a blocking temperature around $410 \mathrm{~K}$ can be estimated. This value is consistant with $T=435 \mathrm{~K}$ being suf- ficient in defining a homogeneous pinning direction (see Fig. 2).

However, $T_{B}$ depends on several parameters, for example, the ratio between the magnetic AFM anisotropy and the exchange coupling strength, and is therefore not a completely intrinsic material property. A much more natural parameter describing an AFM is $T_{N}$, which in principle can also be determined from FM/AFM exchange coupling experiments. An AFM looses its ability to pin an FM at $T_{B}$; however, the AFM/FM interaction does not vanish. As long as an AFM order exists, magnetic interface interactions will also remain, which will manifest themselves in enhanced $H_{C}$ 's because the interface exchange interaction induces irreversible magnetization changes at the interface of the AFM. By inspecting Fig. 3 we notice that $H_{C}$ decreases strongly up to RT and then levels off to a value which is an order larger than $H_{C}$ found in $\mathrm{Cu}$ covered Co. Thus, $H_{C}$ does not follow the collapse observed in $H_{E}$, indicating that $T_{N}$ does not coincide with $T_{B}$ and is certainly higher than $410 \mathrm{~K}$. Unfortunately, the exact value of $T_{N}$ was not accessible in our experiments.

Nevertheless we can conclude that the in the present study e-fct Mn is an AFM with a reasonably high $T_{N}$ exceeding RT. This has to be compared with $\alpha$-Mn, the thermodynamically stable phase of $\mathrm{Mn}$, where AFM order is only observed below $100 \mathrm{~K}$. From an extrapolation of the magnetic properties of high-temperature quenched $\mathrm{Mn}$ rich $X_{y} \mathrm{Mn}_{1-y}(X=\mathrm{Cu}, \mathrm{Fe}, \mathrm{Ni}, \mathrm{Pd}, \ldots)$ bulk crystals to $y=0$, it can be inferred that fcc or fct $\mathrm{Mn}$ should have a $T_{N}$ between 500 and $540 \mathrm{~K} .^{16}$ This value would be consistant with our results. More dedicated experiments should clarify this in the near future.

The importance of the fct $\mathrm{Mn} / \mathrm{Co}$ rests in its striking simplicity. Both AFM and FM are single crystalline with a simple structure, are made of single elements, are coherently grown, and possess a well-defined interface. These properties make comparative theoretical and experimental studies attractive and feasible, which open opportunities to gain deeper insights into the still incompletely understood exchange anisotropy phenomenon.

${ }^{1}$ J. Nogués and I. K. Schuller, J. Magn. Magn. Mater. 192, 203 (1999).

${ }^{2}$ A. E. Berkowitz and K. Takano, J. Magn. Magn. Mater. 200, 552 (1999).

${ }^{3}$ S. A. Wolf, D. D. Awschalom, R. A. Buhrman, J. M. Daughton, S. von Molnár, M. L. Roukes, A. Y. Chtchelkanova, and D. M. Treger, Science 294, 1488 (2001).

${ }^{4}$ V. Skumryev, S. Stoyanov, Y. Zhang, G. Hadjipanayis, D. Givord, and J. Nogués, Nature (London) 423, 850 (2003).

${ }^{5}$ R. Jungblut, R. Coehoorn, M. T. Johnson, J. aan de Stegge, and A. Reinders, J. Appl. Phys. 75, 6659 (1994).

${ }^{6}$ F. Offi, W. Kuch, and J. Kirschner, Phys. Rev. B 66, 064419 (2002).

${ }^{7}$ J. Hafner and D. Spišák, Phys. Rev. B 72, 144420 (2005).

${ }^{8}$ J. T. Kohlhepp and W. J. M. de Jonge (unpublished).

${ }^{9}$ M. Wuttig, B. Feldmann, J. Thomassen, F. May, H. Zillgen, A. Brodde, H. Hannemann, and H. Neddermeyer, Surf. Sci. 291, 14 (1993).

${ }^{10}$ J. P. Pendry, Low Energy Electron Diffraction (Academic, London, 1974).

${ }^{11}$ M. P. Seah and W. A. Dench, Surf. Interface Anal. 1, 2 (1979).

${ }^{12}$ H. Wieldraaijer, W. J. M. de Jonge, and J. T. Kohlhepp, Phys. Rev. B 72, 155409 (2005).

${ }^{13}$ J. T. Kohlhepp and W. J. M. de Jonge, Phys. Rev. Lett. 96, 237201 (2006).

${ }^{14}$ C. M. Schneider, P. Bressler, P. Schuster, J. Kirschner, J. J. de Miguel, and R. Miranda, Phys. Rev. Lett. 64, 1059 (1990).

${ }^{15}$ P. Krams, F. Lauks, R. L. Stamps, B. Hillebrands, and G. Güntherodt, Phys. Rev. Lett. 69, 3674 (1992).

${ }^{16}$ Y. Endoh and Y. Ishikawa, J. Phys. Soc. Jpn. 30, 1614 (1971). 